\title{
ASSESSMENT OF 2D-DIC STOCHASTIC PATTERNS
}

\author{
Jakub Antoš*, VÁClaV NeŽerka, Michael Somr \\ Czech Technical University in Prague, Faculty of Civil Engineering, Thákurova 7, 16629 Prague 6, Czech \\ Republic \\ * corresponding author: jakub.antos@fsv.cvut.cz
}

\begin{abstract}
Cost of experimental testing of materials and structures subjected to mechanical loading often constitutes a significant portion of a project budget. Therefore a collection of data in a maximum possible amount is desirable. Extensometers and strain-gauges attached to the specimen surface often fail and cannot provide full-field information about the development of displacements and strains. Digital Image Correlation (DIC) is capable of providing such information. Unsuitable texture or artificially applied pattern, essential for DIC analysis, can spoil the DIC outcomes completely. An additional investment into preparation of new experimental testing can be avoided with the use of tools employing algorithms for stochastic pattern assessment. The development of such algorithms and their implementation into an open-source DIC software is the goal of the presented research.
\end{abstract}

KEYWORDS: digital image correlation, stochastic pattern, full-field displacement.

\section{INTRODUCTION}

The measurement of strains and displacements is utilized when investigating behavior of materials or specimens in laboratories, but also for assessment of existing structures. Traditionally, contact measurement techniques have been used, but these introduce challenges in terms of accuracy, require sufficiently flat surface, and their attachment requires a lot of effort. The group of contact methods includes strain gauges, extensometers, or linear variable differential transformers (LVDTs) [1].

Contact methods techniques have a few drawbacks compared to optical full field monitoring: the strain must be within a certain range and is averaged over the strain-gauge length, the measurement is accomplished at discrete locations, and the investigated surface must be smooth enough to attach the gauge.

Quite recently alternatives to non-contact measurement have been developed. These include e.g., laser vibrometry, laser range finders, optical correlation methods, interferometry, moiré, or photo/videogrammetry [2, 3]. Optical measurements, such as Digital Image Correlation (DIC), are capable of capturing even the most complex deformation until the ultimate material failure [3, 4]. Because of that reason, optical methods became accepted in the field of experimental solid mechanics. The availability of compact and sensitive light sources, detectors, optical components, and powerful computers have been promoting the use of DIC in both commercial and academic research. The full-field data can be easily processed and provides data valuable for engineers who are used to employing finite element programs in their calculations.

\section{Digital Image Correlation}

DIC became indispensable to monitoring and analyzing a development of displacement or strain fields over time by matching stochastic pattern of deformed images with respect to a reference one [5]. The method is capable of capturing strain localization on a surface of a loaded structure (such as plasticity or cracking) in any direction. As DIC tools for 3D analysis emerge [6], the method is becoming more versatile and widely used in the industry and research.

DIC is not only limited to the relative measurement of discrete points as conventional contact methods and is frequently more accurate than monitoring by means of extensometers or strain-gauges that often suffer from imperfect attachment to the measured surface. Moreover, the use of DIC can significantly reduce the cost of the experiment. DIC reached its maturity during last decade owing it to the fast and successful development and elaboration of computers, digital cameras and specialized software for image processing. DIC has even been characterized as "a major development in the art of experimental mechanics" [7]. The boom of DIC has been reflected in the emergence of new commercial and open source software to perform DIC with differently optimized correlation functions and calculation methods, most recently summarized by Pan et al. [8].

\subsection{DIC Algorithms}

DIC utilizes a correlation algorithm to obtain displacements and subset deformations by identifying areas of matching grey-scale values between the speckle pattern in each subset of deformed and underformed (reference) images (Figure 1). To facilitate the correlation, a stochastic speckle pattern must be applied (if not present naturally) to a specimen surface in order to provide a random grey-level variations at 
the sufficient quality of which is fundamental to the precision of the measured displacement data. It is well established that the trade-off between the data resolution and precision is affected by the quality of a pattern.

To perform DIC, each image must be divided into a grid of interrogation cells, or subsets, containing a finite number of pixels. Resolution of the data is maximized by reducing the size of the subsets, but as the interrogation cell size decreases, the uncertainty in the strain measurement increases due to a reduction in the number of features to track within the subset [9]. Therefore, the resolution and accuracy of the displacement and deformation fields are limited by the total number of pixels within the images. The center position of each target subset is obtained through searching the peak position of the correlation coefficient field and its deformation is calculated using an iterative approach [10, 11.

DIC has traditionally utilized subset-based local approach [5] that has been very well developed and widely used in commercial or academic DIC packages. However, there are certain drawbacks that make the method useless for some applications, such as experiments producing high strain gradients [12]. The reason is non-continuity of the displacement field, as the displacements of the subsets are sought separately. Therefore smoothing of the noisy displacement field is required [8, 9, 11. A local polynomial smoothing over a rectangular area (strain window) is usually employed [8]. The amount of smoothing is controlled by the subset size, step size, and strain window size. The results are then highly dependent on setting these parameters by a user [13].

An alternative global approach has been developed quite recently based on the procedures used in the Finite Element Method (FEM) [14, 15]. Using the global DIC approach, a complete element mesh is tracked on images, ensuring $C^{0}$-continuity ${ }^{1}$ resulting in elimination of noise in strain fields. This method was initially proposed by Cheng et al. [16] and later Besnard et al. developed the Q4-DIC [17] with implementation of a bilinear (Q4) or quadratic (Q8) mesh. The global algorithms may be refined using user experience, or using adaptive mesh refinement [18].

\subsection{DIC ACCURACY}

The accuracy of DIC is an issue frequently discussed by both, theoreticians and experimentalists [19, 20]. Although originally restricted to the size of one-pixel, various sub-pixel techniques have been proposed and used to improve the method accuracy. The most popular techniques include curve-fitting, gradient-based and Newton-Raphson algorithms. The last two cases are based on the identification of the parameters that define the mapping of a subset of pixels in order to

\footnotetext{
${ }^{1} C^{i}$ differentiability class corresponds to the existence of $i$ derivatives of a function. The class $C^{0}$ consists of all continuous functions.
}

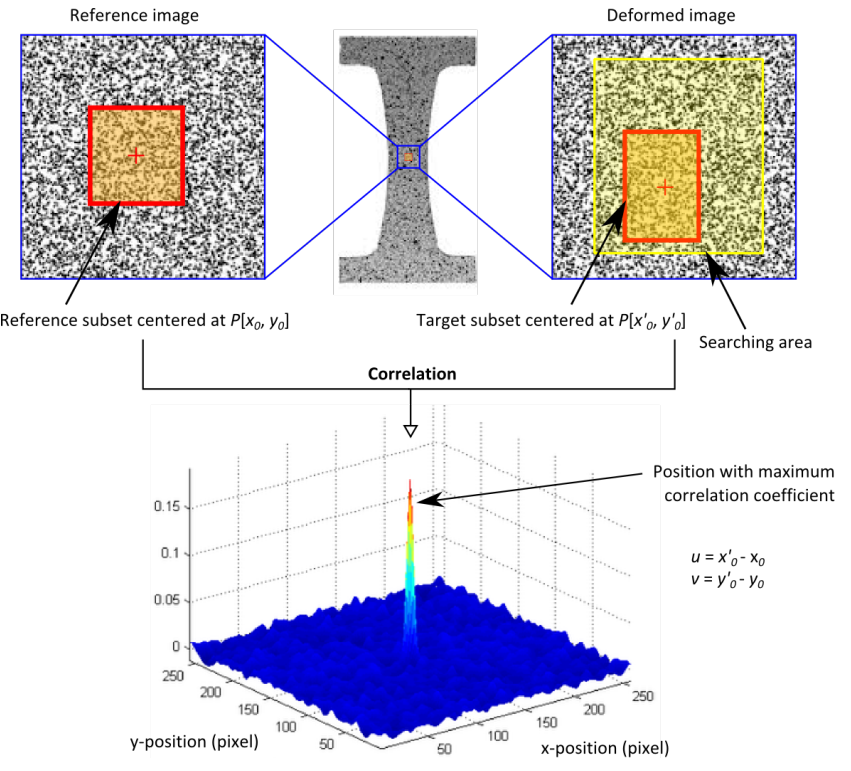

Figure 1. Example of the subset tracking procedure using DIC.

maximize the image correlation. The results show that the Newton-Raphson approach is considered as the most accurate and stable. Other interesting approaches such as genetic algorithms, finite element interpolations and B-splines are reported in [8] but they generally appear to have a lower performance. Bing et al. 21] compared performance of three most used sub-pixel techniques by simulating image distortions, demonstrating the suitability of Newton-Rapson approach.

Besides the choice of the most suitable DIC algorithm, other factors substantially influence the DIC accuracy. The measurement errors may be classified into two categories: (i) systematic experimental errors such as bad calibration and setting of image acquisition system and (ii) errors produced by correlation algorithm.

The experimental errors are related to variations in illumination and the quality of the acquisition system, i.e., the noise during the acquisition and digitalization, image distortion, or position of the camera with respect to the monitored surface. The errors related to the correlation algorithms arise from inappropriate selection of correlation methods or input parameters, such as subset size, correlation function, sub-pixel algorithm, shape function, or interpolation scheme.

\section{Stochastic Pattern Assessment}

A majority of research into the accuracy of DIC is focused on correlation algorithms and processing parameters (recall Section 2.2), such as subset size [22, shape function selection [23, or methods of obtaining sub-pixel accuracy [24]. Less attention has been paid to the effect of the quality of the speckle pattern. It is important for the artificial pattern or natural texture to be adjusted in accordance with the expected displacement field in order to maximize measurement 
accuracy, as speckles can be both too large and too small for the particular measurement [25]. Quantitative error analysis 26-29] shows that the measurement error depends crucially on the presence of large intensity gradients within the pattern. Therefore, the technique in most cases requires a specimen preparation procedure to enhance the surface texture, since the natural one does not provide a required quality in terms of intensity gradients and speckle-size distribution.

Pattern assessment has been discussed by a few authors [5, 30, 31, offering a number of methods to evaluate the quality of applied patterns. Pan et al. 32 presented a number of assessment criteria based upon the local subset intensity gradients [20] and the global mean intensity gradient (MIG) throughout the image. The latter criteria showed a good agreement to results obtained numerically. The benefit of applying the global criteria is that these are astraightforward. High MIG values typically result in smaller bias and less dispersion in DIC measurements. On the other hand, Crammonde et al. [33] objected that global parameters, such as MIG, are not sufficient to evaluate strain accuracy. Based on their findings, simple means of a randomness assessment is to employ the outcomes of the Shannon entropy theory [34.

Cintrón et al. 35] demonstrated that the speckle size should range from $2 \times 4$ px for a high accuracy in the strain measurement. The speckle size should have a lower limit according to the study by Sutton et al. 5] of $3 \times 3 \mathrm{px}$.

In the presented study, various patterns produced by the spraying white / black dots on a contrast background were investigated. In addition, natural textures of various materials were studied as well, to demonstrate their performance. The knowledge of a texture suitability is essential when monitoring existing structures where application of artificial patterns is not possible. To eliminate the impact caused by image acquisition system ( $\mathrm{such}$ as the distortion of the camera lens, lighting variation, etc.) during the experiment, numerical approach was utilized to investigate the relationship between Shannon entropy, mean intensity gradient, values of correlation coefficient, and error in DIC measurements. The explanation of the mentioned parameters is provided next.

\subsection{Normalized Cross-Correlation}

Correlation between two signals (also reffered to as cross-correlation) is a standard approach to feature detection and image registration [36-38]). The normalized form of correlation takes into account intensities relative to the highest one, thus eliminating the sensitivity to changes in illumination intensity. However, it does not have a correspondingly simple and efficient frequency domain expression. For this reason, normalized cross-correlation must be computed in a spatial domain and fast spatial domain matching methods had to be developed. Detailed description of cross-correlation equation is beyond the scope of this article. Reader can find it in Barnea et al. [39.

\subsection{Mean Intensity Gradient (MIG)}

MIG was proposed by Pan et al. 32 as a global parameter for evaluating the quality of a speckle pattern over the entire domain. Both, a mean bias error and standard deviation of measured displacements are influenced by the MIG of the speckle pattern. The speckle pattern with a large MIG is supposed to produce small displacement measurement errors. MIG is defined as

$$
\delta_{f}=\sum_{i=1}^{W} \sum_{j=1}^{H}\left|\nabla \mathrm{f}\left(x_{i j}\right)\right| \frac{1}{W \times H},
$$

where $W$ and $H$ are the image width and height in pixels, $\left|\nabla \mathrm{f}\left(x_{i j}\right)\right|=\sqrt{f_{i}\left(x_{i j}\right)^{2}+f_{j}\left(x_{i j}\right)^{2}}$ is the modulus of the local intensity gradient. $f_{i}\left(x_{i j}\right)$ and $f_{j}\left(x_{i j}\right)$ are the $i$ - and $j$-directional intensity derivatives at pixel $\left(x_{i j}\right)$ respectively, which can be computed using a central difference algorithm.

\subsection{ShannOn EnTROPy}

A high Shannon entropy value indicates a high level of texturing, or broadness in the greyscale distribution of the image, beneficial for maximizing the correlation function peak when a correct match has been found. The Shannon entropy parameter is defined as [40]

$$
\Psi=\sum_{i=1}^{W} \sum_{i=j}^{H} f\left(x_{i j}\right) \log \left(f\left(x_{i j}\right)\right) .
$$

\section{Assessment of Patterns And TeXTuRES}

Randomly distributed speckles must not exhibit any preferential directions. High contrasts are required to provide sharp peaks in the correlation function. To test this requirement, initial synthetic tests on samples of $400 \times 400 \mathrm{px}$ were performed on six model patterns (Figure 2). The patterns / textures were tested with respect to four quality indicators: value of cross-correlation coefficient for two different subset sizes $(10 \times 10 \mathrm{px}$ and $30 \times 30 \mathrm{px})$, Shannon entropy, and MIG. The summary of the investigated patterns and textures is provided in Table 1 .

Based on the values of normalized cross-correlation functions presented in the form of surface plots in Figures 3 and 4 , it can be concluded that the correlation function peaks can be highlighted by setting bigger subset size since these contain more distinct features. Especially in the case of small subset size, $10 \times 10 \mathrm{px}$, the correlation peaks on coarse textures could not be clearly identified. This could lead to huge errors of DIC measurements in the case of significantly distorted images. However, the findings cannot be generalized, since in some patterns the cross-correlation correlation function does not exhibit any distinct peak 


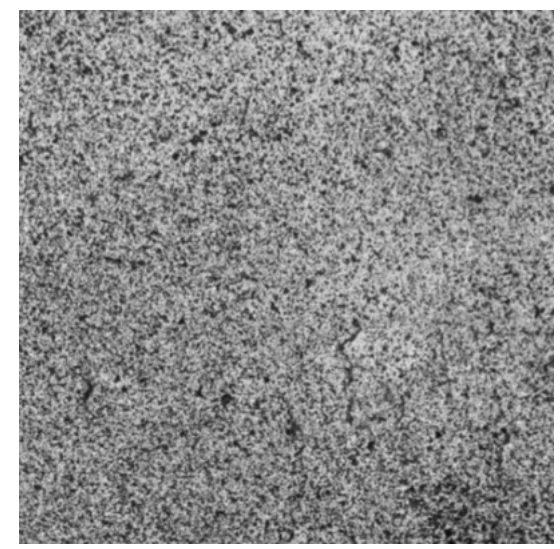

(A) . fine spray pattern

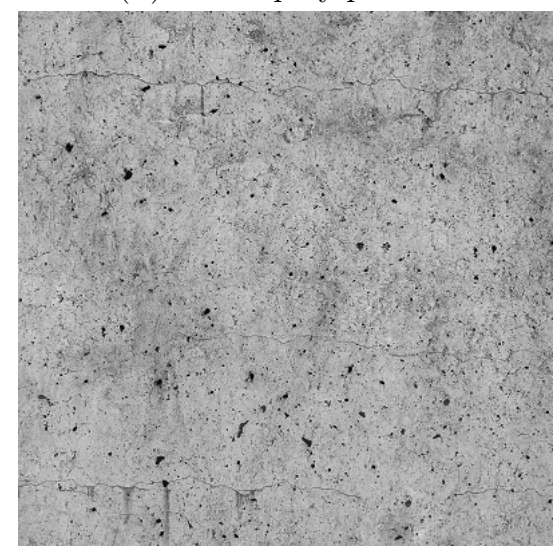

(D) . natural texture of concrete

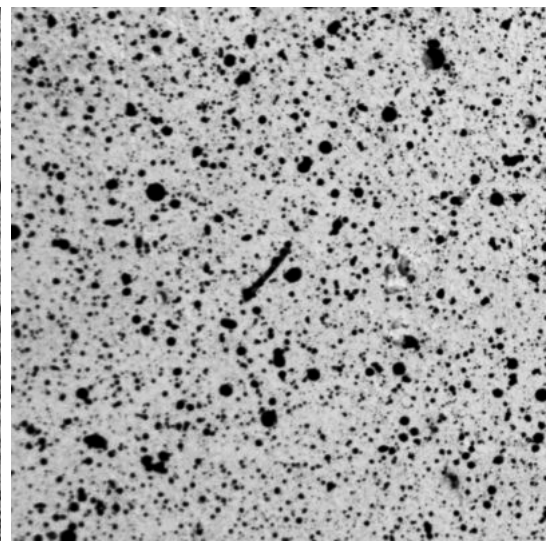

(в) . coarse air-brush pattern

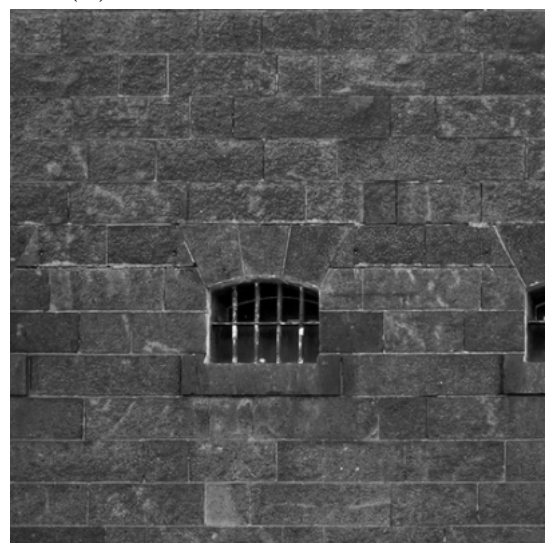

(E) . masonry wall

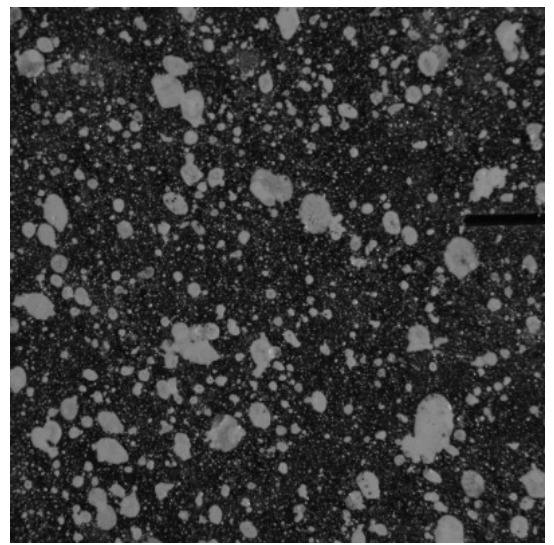

(C) . white sprayed dots

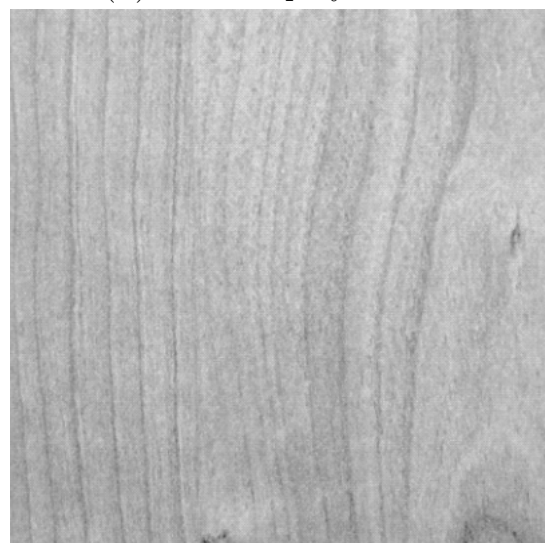

(F) . texture of wood

FiguRE 2. Investigated artificial patterns (top) and natural textures (bottom).

in for small subsets, while correlation of the larger one results in unique match and low value of mean correlation coefficient (cf. Figures 3(b,f), 4 (b,f), and Table 1). Therefore, the pattern assessment based on the the mean value of the cross-correlation function must be carried out for any subset size independently. Moreover, a statistical information about number of peaks in the form of a decay in the power law distribution would provide more accurate description.

Based on observation of distribution of correlation coefficient a power law distribution can be assumed (Figure 5, 6). The power law function is defined by equation $y_{p}=x_{p}^{-\alpha}$, where $\alpha$ is the decay parameter which provides information about the distribution of correlation coefficient over the investigated domain. Larger $\alpha$ indicates smaller amount of unwanted correlation peaks which is desirable.

It is also clear that neither Shannon entropy nor MIG are capable of providing clear indication of the pattern quality since these two are not in agreement once the pattern is, relative to other ones, regarded as superior to others based on Shannon entropy, MIG provides completely different results, see Table 1 . Therefore, new more universal criteria taking into account size, contrast, and size-distribution of speckles should be proposed.

\section{Virtual Stretching of Patterns and Textures}

The artificial patterns and natural textures were tested in the proof-of-concept experiments carried out virtually on a computer by prescribing a constant deformation. Special attention was paid to the ability of the pattern to provide high-accuracy measurements at deformations reaching $5 \%$, at which poor patterns fail [41].

The evaluation and testing of patterns was performed using own MATLAB scripts by placing virtual extensometers at the edges of the tested samples. In this initial study the images containing 8-bit speckle patterns were virtually stretched up to $5 \%$ in 10 steps. Image sequences were then evaluated in open-source 2D-DIC software package Ncorr [10] with the same setting of parameters. Subset sizes of $10 \mathrm{px}$ and $30 \mathrm{px}$ were chosen to demonstrate the correlation between the mean correlation coefficient mentioned in the Section 4 and measurement errors.

As the number of speckles within the patterns increased, the measurement errors decreased. Moreover, larger sized speckles provided lower error than patterns with smaller speckles. Global pattern quality parameters were discussed and Shannon entropy was used as an example to demonstrate that the global measures are not sufficient to assess the quality and 


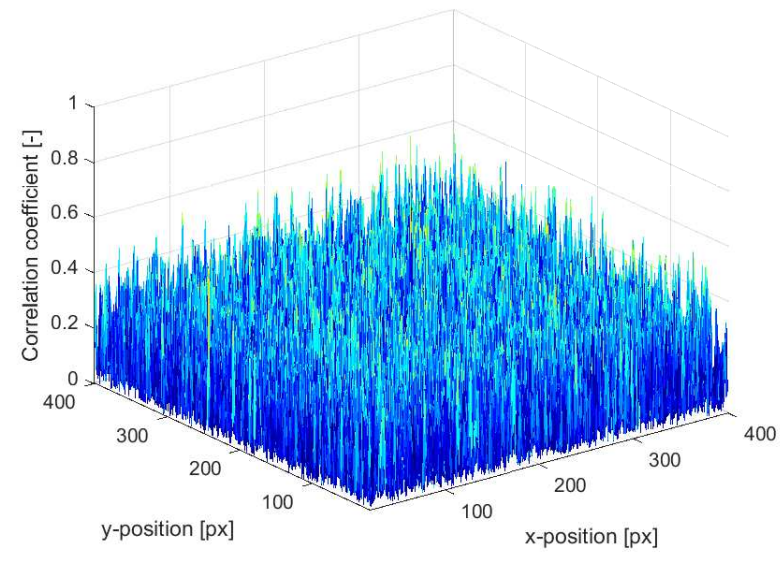

(A) . fine spray pattern

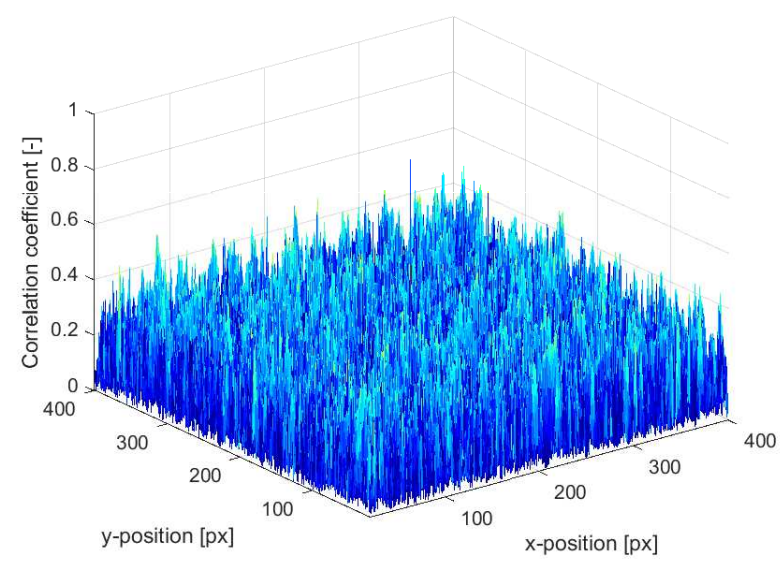

(C) . white sprayed dots

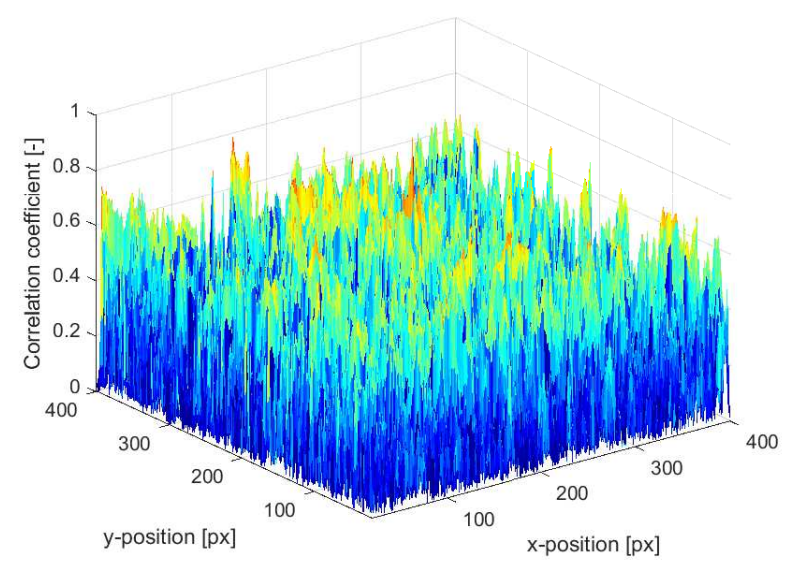

(E) . masonry wall

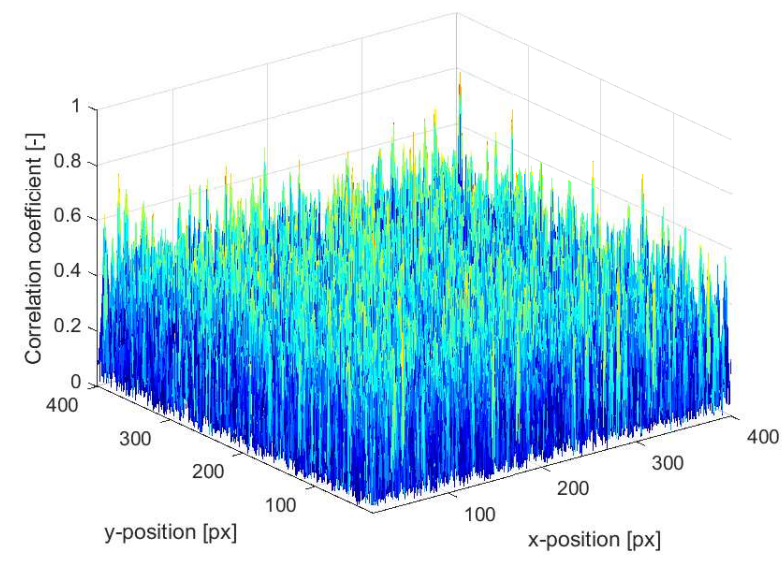

(В) . coarse air-brush pattern

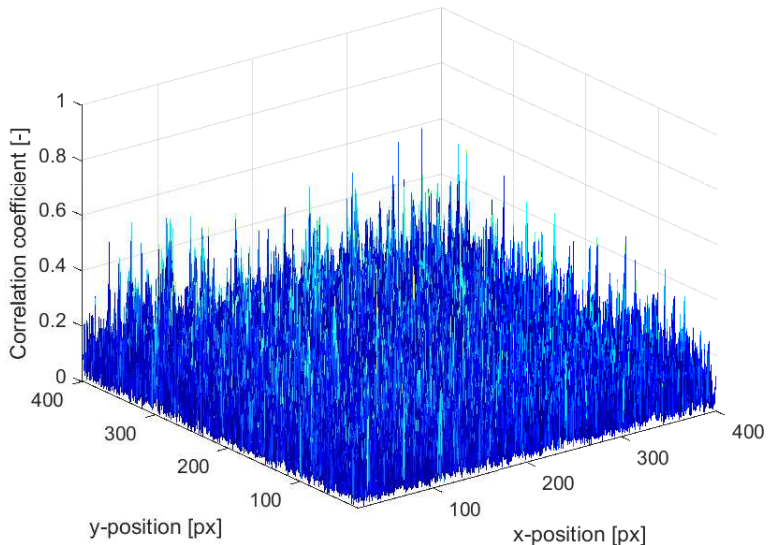

(D) . natural texture of concrete

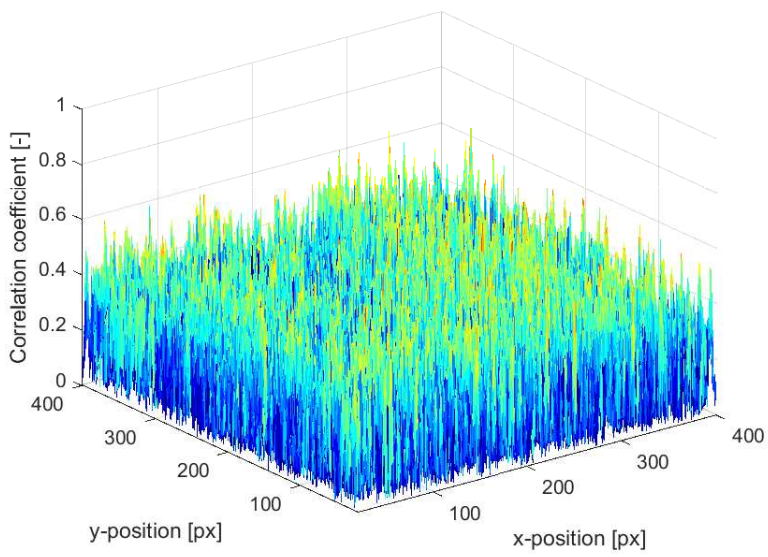

(F) . texture of wood

Figure 3 . Correlation function values for $10 \times 10$ px subset size. 


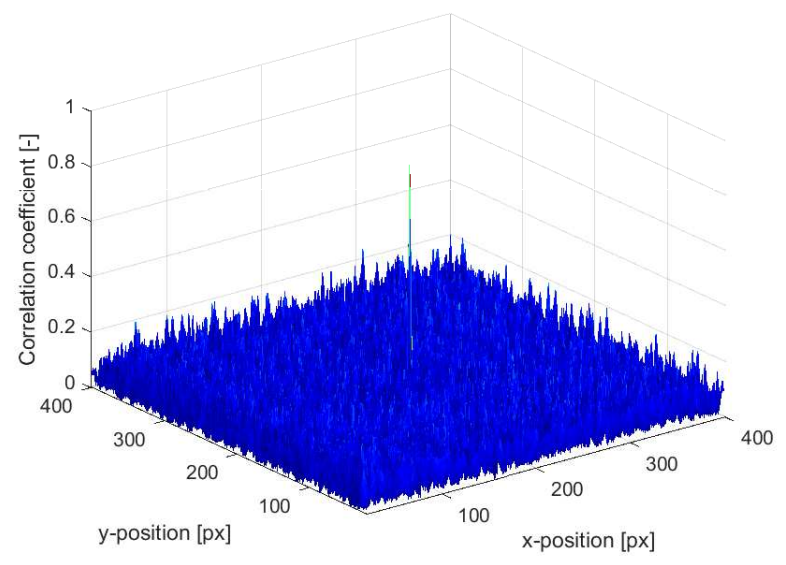

(A) . fine spray pattern

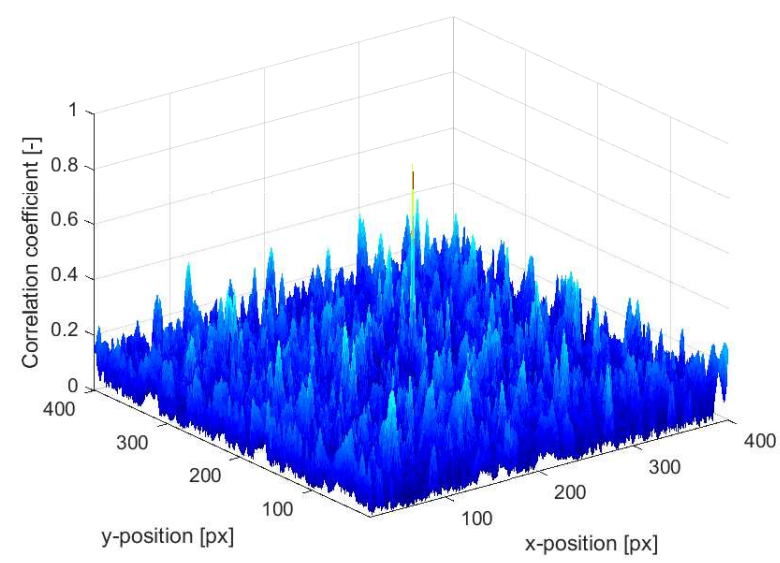

(C) . white sprayed dots

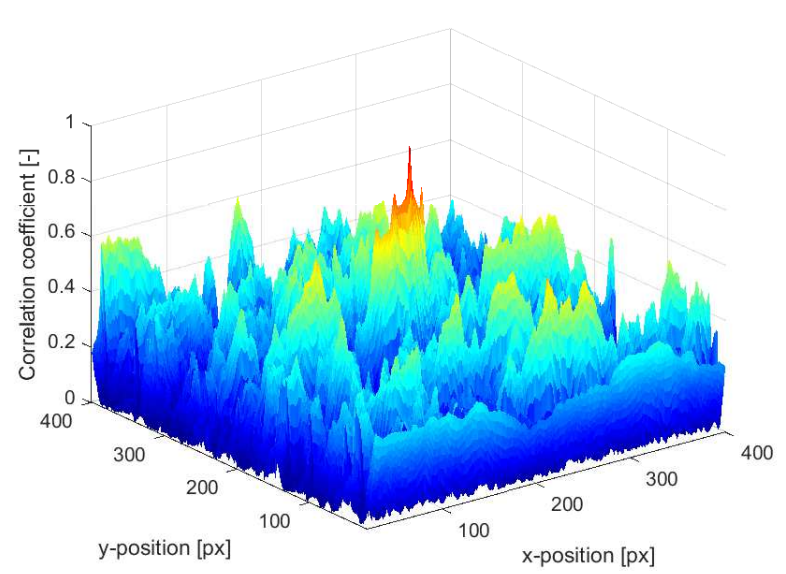

(E) . masonry wall

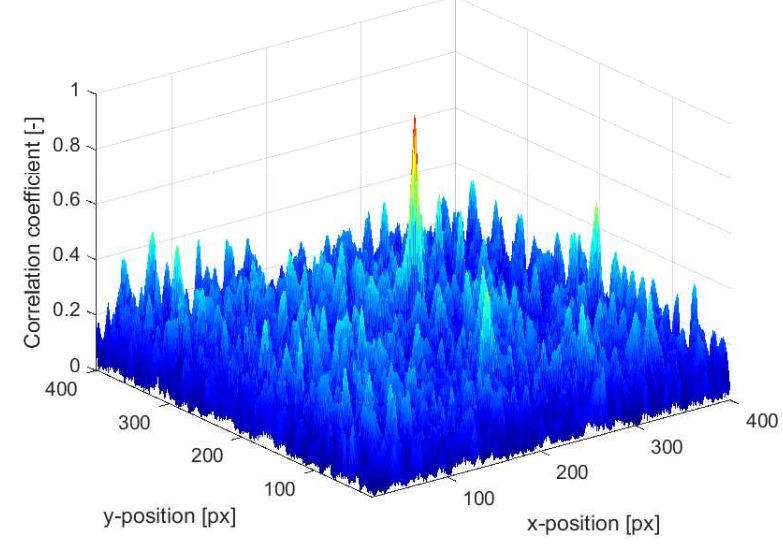

(в) . coarse air-brush pattern

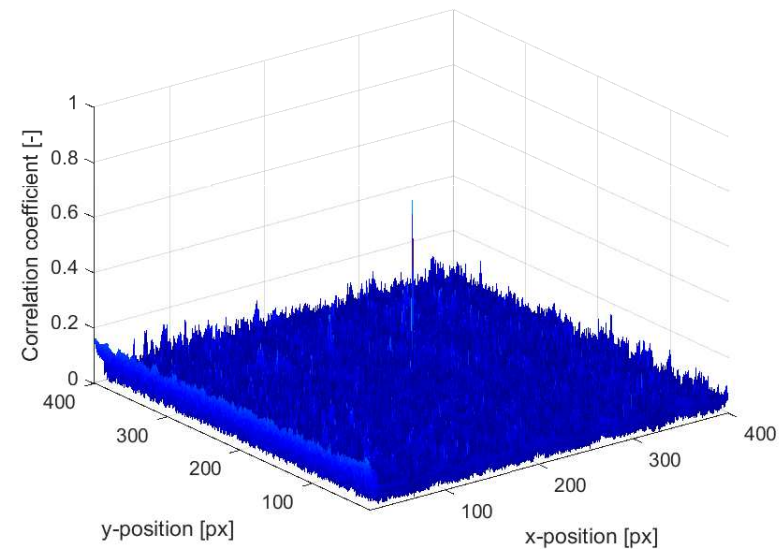

(D) . natural texture of concrete

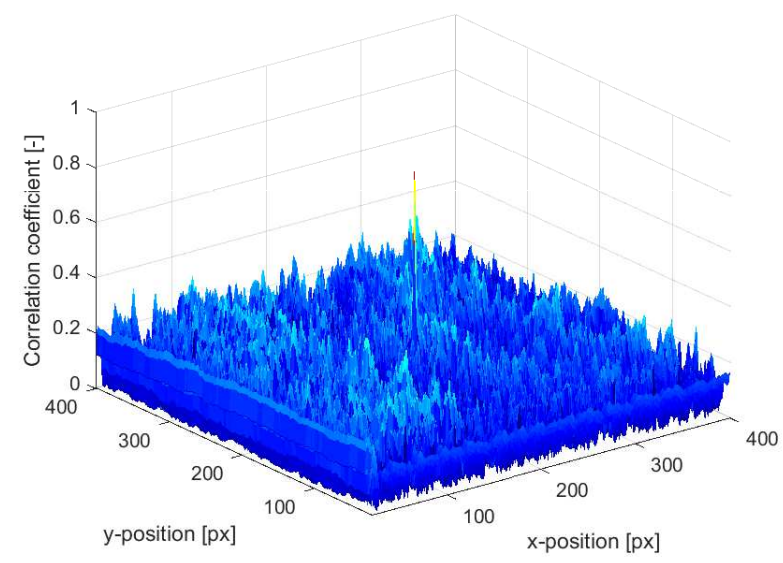

(F) . texture of wood

Figure 4. Correlation function values for $30 \times 30 \mathrm{px}$ subset size. 


\begin{tabular}{c|cc|cc|c|c} 
& \multicolumn{2}{|c|}{$\overline{\gamma\left(x_{i j}\right)}$} & \multicolumn{2}{|c|}{$\alpha$} & $\Psi$ & $\delta_{f}$ \\
Sample & $10 \times 10$ & $30 \times 30$ & $10 \times 10$ & $30 \times 30$ & & \\
\hline Fine spray pattern (a) & 0.152 & 0.054 & 0.847 & 0.959 & 5.524 & 121.182 \\
Coarse air-brush pattern (b) & 0.190 & 0.107 & 1.009 & 1.046 & 5.413 & 127.614 \\
White spray dots (c) & 0.089 & 0.138 & 1.148 & 0.997 & 4.972 & 82.198 \\
Natural texture of concrete (d) & 0.090 & 0.039 & 1.027 & 0.975 & 6.292 & 75.849 \\
Masonry wall (e) & 0.200 & 0.187 & 0.879 & 0.939 & 4.250 & 50.914 \\
Texture of wood (f) & 0.208 & 0.097 & 0.878 & 0.981 & 5.012 & 29.027
\end{tabular}

TABLE 1. Mean correlation coefficient, $\overline{\gamma\left(x_{i j}\right)}$, power law distribution decay parameter $\alpha, \Psi$, and $\delta_{f}$ for tested patterns / textures and different size of subsets.

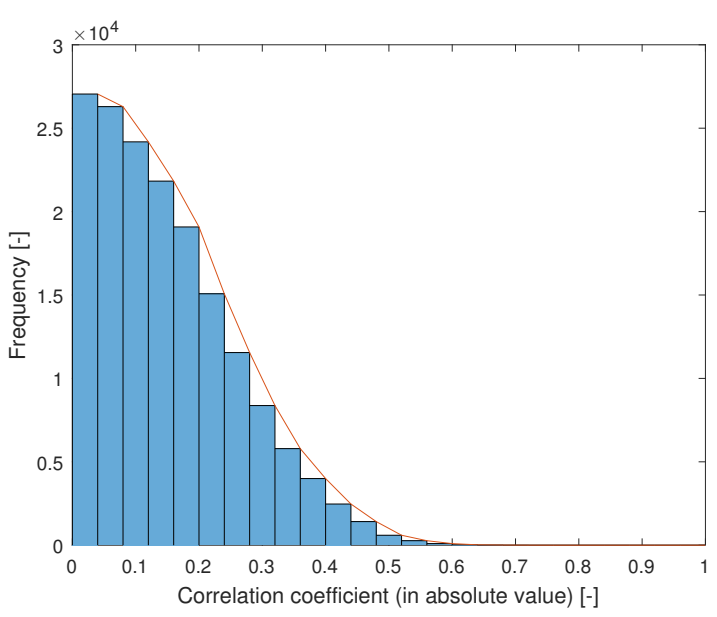

(A) . subset $10 \times 10 \mathrm{px}(\alpha=0.847)$

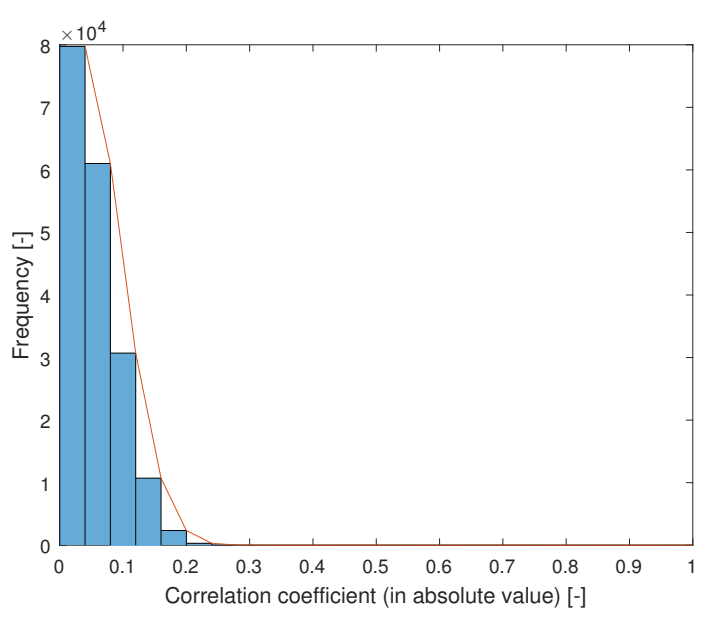

(B) . subset $30 \times 30 \mathrm{px}(\alpha=0.959)$

FiguRE 5. Distribution of correlation coefficient, fine spray pattern.

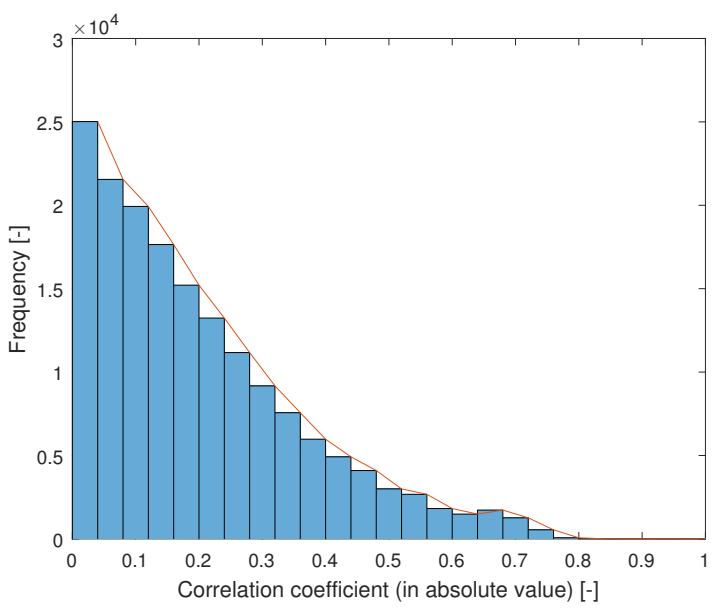

(A) . subset $10 \times 10 \mathrm{px}(\alpha=0.879)$

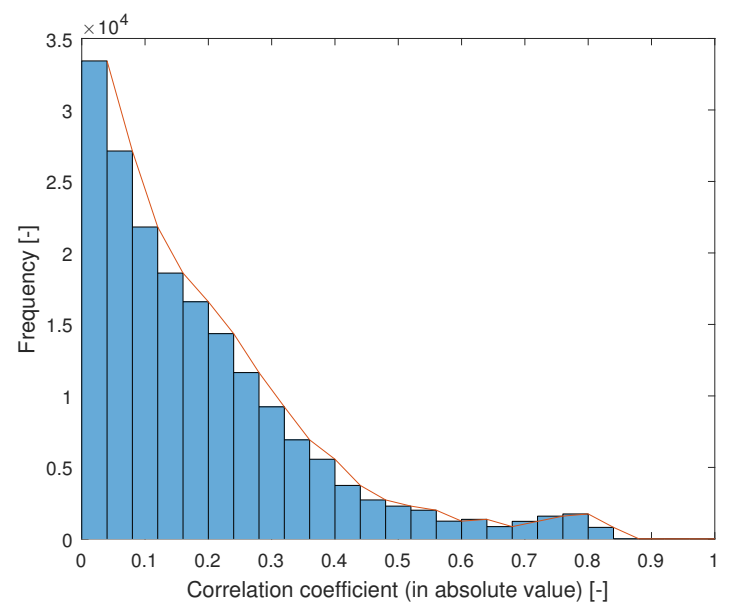

(B) . subset $30 \times 30 \mathrm{px}(\alpha=0.939)$

FIgURE 6. Distribution of correlation coefficient, masonry wall. 


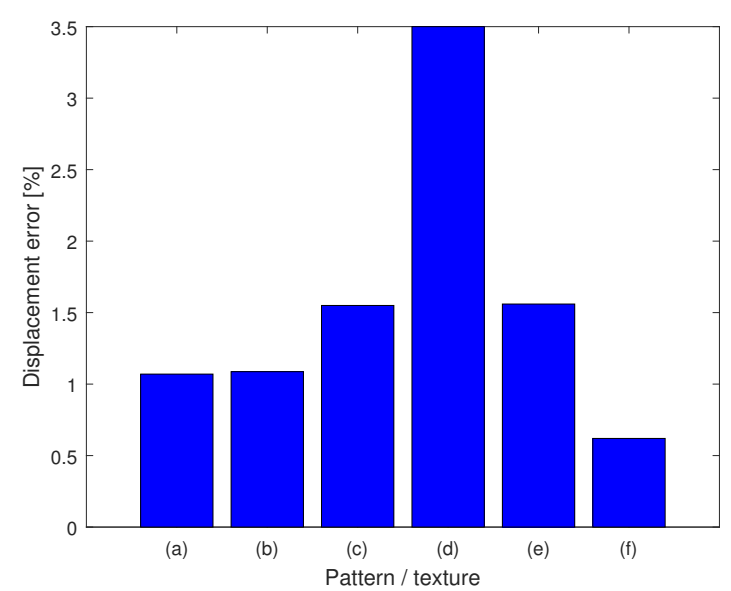

(A) . $1 \%$ deformation

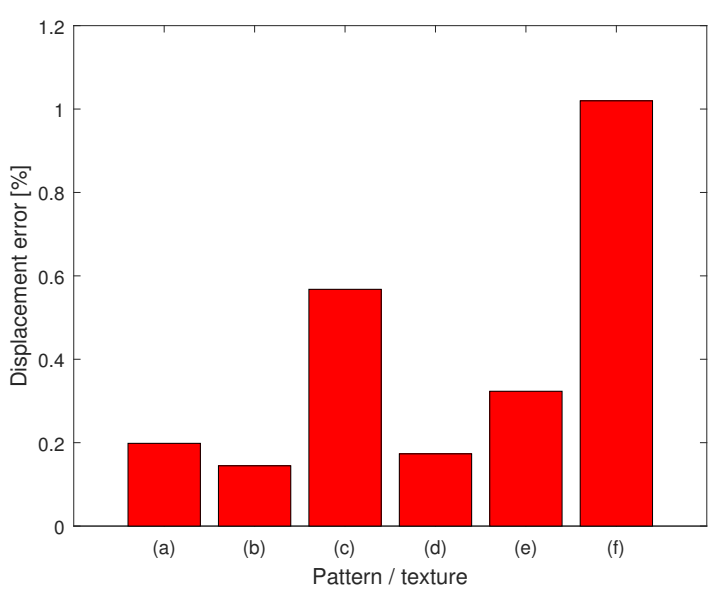

(B) . $5 \%$ deformation

Figure 7. Displacement error for $10 \times 10$ px subset size.

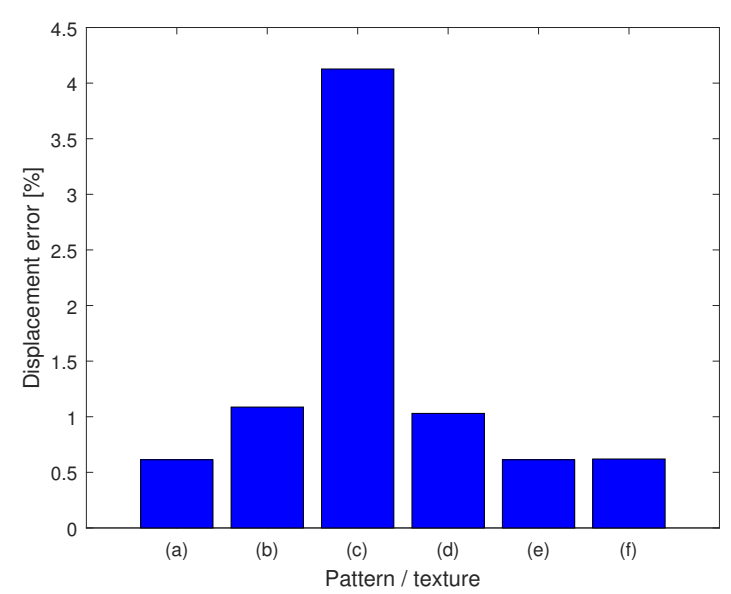

(A) $.1 \%$ deformation

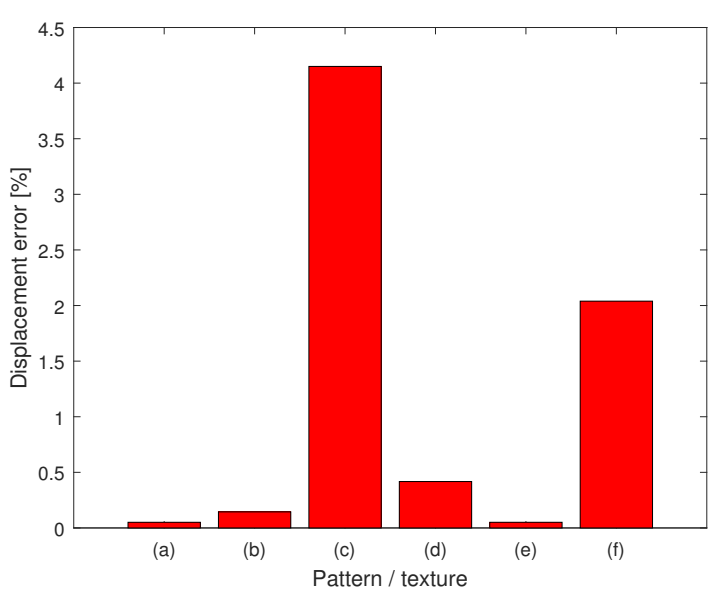

(B) . $5 \%$ deformation

Figure 8 . Displacement error for $30 \times 30$ px subset size.

properties of the patterns. This is illustrated by comparing errors at different stretching magnitude for different speckle sizes (Figure 7 and 8 ).

\section{Conclusions}

Based on the research of Crammond et al. [33] and outcomes of the presented study it can be concluded that multi-criteria approach has to be addressed. So far it appears that a combination of Shannon entropy and evaluation of decay in power law distribution of correlation coefficient values provide the most accurate estimate of a pattern performance. Moreover, the speckle-size histogram analysis will be carried out in future to provide new, hopefully more reliable, assessment criteria.

\section{ACKNOWLEDGEMENTS}

This work was financially supported by Czech Technical University in Prague - SGS project SGS17/043/OHK1/1T/11.

\section{REFERENCES}

[1] C. A. Sciammarella, F. M. Sciammarella. Experimental Mechanics of Solids. Wiley-Blackwell, 2012. DOI:10.1002/9781119994091.

[2] T. Yoshizawa (ed.). Handbook of Optical Metrology. Informa UK Limited, 2015. DOI:10.1201/b18328.

[3] K. H. Laermann (ed.). Optical Methods in Experimental Solid Mechanics. Springer Nature, 2000. DOI:10.1007/978-3-7091-2586-1.

[4] J. Périé, S. Calloch, C. Cluzel, F. Hild. Analysis of a multiaxial test on a c/c composite by using digital image correlation and a damage model. Experimental Mechanics 42(3):318-328, 2002. DOI:10.1007/bf02410989.

[5] H. Schreier, J. Orteu, M. Sutton. Image Correlation for Shape, Motion and Deformation Measurements. Springer Nature, 2009. DOI:10.1007/978-0-387-78747-3.

[6] V. Nežerka, J. Antoš, T. Sajdlová, P. Tesárek. Use of open source DIC tools for analysis of multiple cracking in fiber-reinforced concrete. Applied Mechanics and Materials 827:336-339, 2016. DOI:10.4028/www.scientific.net/amm.827.336. 
[7] F. Chiang. Super-resolution digital speckle photography for micro/nano measurements. Optics and Lasers in Engineering 47:274-279, 2009.

[8] B. Pan, K. Qian, H. Xie, A. Asundi. Two-dimensional digital image correlation for in-plane displacement and strain measurement: a review. Measurement Science and Technology 20(6):062001, 2009. DOI:10.1088/0957-0233/20/6/062001

[9] K. Triconnet, K. Derrien, F. Hild, D. Baptiste. Parameter choice for optimized digital image correlation. Optics and Lasers in Engineering 47(6):728-737, 2009. DOI:10.1016/j.optlaseng.2008.10.015

[10] J. Blaber, B. Adair, A. Antouniou. Ncorr: Open-source $2 \mathrm{~d}$ digital image correlation matlab software. Experimental Mechanics 55:1105-1122, 2015. DOI:10.1007/s11340-015-0009-1.

[11] B. Pan, A. Asundi, H. Xie, J. Gao. Digital image correlation using iterative least squares and pointwise least squares for displacement field and strain field measurements. Optics and Lasers in Engineering 47(78):865-874, 2009. DOI:10.1016/j.optlaseng.2008.10.014

[12] F. Lagattu, J. Brillaud, M. Lafarie-Frenot. High strain gradient measurements by using digital image correlation technique. Materials Characterization 53(1):17-28, 2004. DOI:10.1016/j.matchar.2004.07.009

[13] T. Proulx (ed.). Application of Imaging Techniques to Mechanics of Materials and Structures, Volume 4. Springer New York, 2013. DOI:10.1007/978-1-4419-9796-8

[14] J. Fish, T. Belytschko. A First Course in Finite Elements. Wiley-Blackwell, 2007. DOI:10.1002/9780470510858

[15] D. L. Logan. A First Course in the Finite Element Method. Chris Carson, 2007.

[16] P. Cheng, M. Sutton, H. Schreier, S. McNeill. Full-field speckle pattern image correlation with b-spline deformation function. Experimental Mechanics 42(3):344-352, 2002. DOI:10.1177/001448502321548445

[17] G. Besnard, F. Hild, S. Roux. "finite-element" displacement fields analysis from digital images: Application to portevin-le châtelier bands. Experimental Mechanics 46(6):789-803, 2006. DOI:10.1007/s11340-006-9824-8

[18] L. Wittevrongel, P. Lava, S. V. Lomov, D. Debruyne. A self adaptive global digital image correlation algorithm. Experimental Mechanics 55(2):361-378, 2014. DOI:10.1007/s11340-014-9946-3

[19] P. J. Withers. Strain measurement by digital image correlation. Strain 44(6):421-422, 2008. DOI:10.1111/j.1475-1305.2008.00556.x

[20] B. Pan, H. Xie, Z. Wang, et al. Study on subset size selection in digital image correlation for speckle patterns. Optics Express 16(10):7037, 2008. DOI:10.1364/oe.16.007037

[21] P. Bing, X. Hui-min, X. Bo-qin, D. Fu-long. Performance of sub-pixel registration algorithms in digital image correlation. Measurement Science and Technology 17(6):1615-1621, 2006. DOI:10.1088/0957-0233/17/6/045
[22] S. Yaofeng, J. H. Pang. Study of optimal subset size in digital image correlation of speckle pattern images. Optics and Lasers in Engineering 45(9):967-974, 2007. DOI:10.1016/j.optlaseng.2007.01.012

[23] H. W. Schreier, M. A. Sutton. Systematic errors in digital image correlation due to undermatched subset shape functions. Experimental Mechanics 42(3):303-310, 2002. DOI:10.1007/bf02410987.

[24] H. W. Schreier. Systematic errors in digital image correlation caused by intensity interpolation. Optical Engineering 39(11):2915, 2000. DOI:10.1117/1.1314593.

[25] P. Hung, A. S. Voloshin. In-plane strain measurement by digital image correlation. Journal of the Brazilian Society of Mechanical Sciences and Engineering 25(3), 2003. DOI:10.1590/s1678-58782003000300001.

[26] Y. Q. Wang, M. A. Sutton, H. A. Bruck, H. W. Schreier. Quantitative error assessment in pattern matching: Effects of intensity pattern noise, interpolation, strain and image contrast on motion measurements. Strain 45(2):160-178, 2009. DOI:10.1111/j.1475-1305.2008.00592.x

[27] M. e. a. Bornert. Assessment of digital image correlation measurement errors: Methodology and results. Experimental Mechanics 49(3):353-370, 2008. DOI:10.1007/s11340-008-9204-7.

[28] J. Dupré, M. Bornert, L. Robert, B. Wattrisse. Digital image correlation: displacement accuracy estimation. EPJ Web of Conferences 6:31006, 2010. DOI:10.1051/epjconf/20100631006

[29] B. Pan, K. Qian, H. Xie, A. Asundi. On errors of digital image correlation due to speckle patterns. In X. He, H. Xie, Y. Kang (eds.), ICEM 2008: International Conference on Experimental Mechanics 2008. SPIE-Intl Soc Optical Eng, 2008. DOI:10.1117/12.839326.

[30] T. Hua, H. Xie, S. Wang, et al. Evaluation of the quality of a speckle pattern in the digital image correlation method by mean subset fluctuation. Optics \& Laser Technology 43(1):9-13, 2011. DOI:10.1016/j.optlastec.2010.04.010

[31] C. Lane, R. L. Burguete, A. Shterenlikht. An objective criterion for the selection of an optimum dic pattern and subset size. In Proceedings of the SEM XI international congress and exposition on experimental and applied mechanics. 2008.

[32] B. Pan, Z. Lu, H. Xie. Mean intensity gradient: An Effective global parameter for quality assessment of the speckle patterns used in digital image correlation. Optics and Lasers in Engineering 48:469-477, 2010.

[33] G. Crammond, S. W. Boyd, J. M. Dulieu-Barton. Speckle pattern quality assessment for digital image correlation. Optics and Lasers in Engineering 51(12):1368-1378, 2013. DOI:10.1016/j.optlaseng.2013.03.014

[34] C. E. Shannon. A mathematical theory of communication. Bell System Technical Journal 27(3):379-423, 1948. DOI:10.1002/j.1538-7305.1948.tb01338.x

[35] R. Cintrón, V. Saouma. Strain measurements with the digital image correlation system vic-2d, report cunees-08-06. Tech. rep., NEES at CU Boulder, 2008. 
[36] O. Duda, E. H. Peter. Pattern classification and scene analysis. Artificial Intelligence 4(2):139-143, 1973. DOI:10.1016/0004-3702(73)90004-0

[37] R. C. Gonzalez, R. E. Woods, B. R. Masters. Digital image processing, third edition. Journal of Biomedical Optics 14(2):029901, 2009. DOI:10.1117/1.3115362.

[38] R. Brunelli, T. Poggio. Face recognition: features versus templates. IEEE Transactions on Pattern Analysis and Machine Intelligence 15(10):1042-1052, 1993. DOI:10.1109/34.254061

[39] D. I. Barnea, H. F. Silverman. A class of algorithms for fast digital image registration. IEEE Transactions on Computers C-21(2):179-186, 1972.

DOI:10.1109/tc.1972.5008923.
[40] D. Lecompte, S. Bossuyt, S. Cooreman, et al. Study and generation of optimal speckle patterns for dic. In Proceedings of the SEM annual conference and exposition on experimental and applied mechanics. 2007.

[41] G. Stoilov, V. Kavardzhikov, D. Pashkouleva. A comparative study of random patterns for digital image correlation. Journal of Theoretical and Applied Mechanics 42:55-66, 2012. 